\title{
Quantum Games
}

\author{
Jens Eisert and Martin Wilkens \\ Institut für Physik, Universität Potsdam, 14469 Potsdam, Germany
}

(August 20, 2018)

In these lecture notes we investigate the implications of the identification of strategies with quantum operations in game theory beyond the results presented in [J. Eisert, M. Wilkens, and M. Lewenstein, Phys. Rev. Lett. 83, 3077 (1999)]. After introducing a general framework, we study quantum games with a classical analogue in order to flesh out the peculiarities of game theoretical settings in the quantum domain. Special emphasis is given to a detailed investigation of different sets of quantum strategies.

PACS-numbers: 03.67.-a, 03.65.Bz, 02.50.Le

\section{INTRODUCTION}

Game theory is the theory of decision making, which provides powerful tools for investigating situations in which several parties make decisions according to their personal interest [1] 4 ]. It gives an account of how the parties would decide in a situation which involves contest, rivalry, or struggle. Such have been found to be relevant to social sciences, biology, or economics. Of particular interest to the theory are games of incomplete information in which the parties may choose their plan of action with complete knowledge of the situation on rational grounds, but without knowing what decision the other parties have actually taken.

One important two player game is the so-called Prisoners' Dilemma [5]. In this game two players - in the following referred to as Alice and Bob - can independently decide whether they intend to "cooperate" or "defect". Being well aware of the consequences of their decisions the players obtain a certain pay-off according to their respective choices. This pay-off provides a quantitative characterisation of their personal preferences. Both players are assumed to want to maximise their individual pay-off, yet they must pick their choice without knowing the other player's decision. Fig. 1 indicates the pay-off of Alice and Bob. The players face a dilemma since rational reasoning in such a situation dictates the players to defect, although they would both benefit from mutual cooperation. As Alice is better off with defection regardless of Bob's choice, she will defect. The game being symmetric, the same argument applies to Bob.

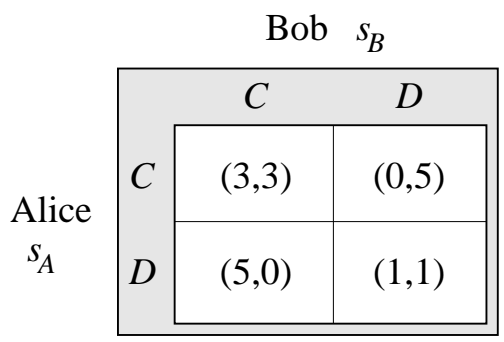

FIG. 1. The pay-off matrix in the Prisoners' Dilemma game. The first entry refers to Alice's pay-off, the second to Bob's. If both players cooperate, they both get 3 units pay-off. If Bob defects and Alice happens to cooperate, he obtains 5 units, while Alice is in the unfortunate situation in which she does not receive any pay-off at all. Bob faces the same situation if he chooses to cooperate while Alice prefers to defect. If both defect, they get only 1 unit pay-off. 
Formally, Alice has two basic choices, meaning that she can select from two possible strategies $s_{A}=C$ (cooperation) and $s_{A}=D$ (defection). Bob may also take $s_{B}=C$ or $s_{B}=D$. The game is defined by these possible strategies on the one hand, and on the other hand by a specification of how to evaluate the pay-off once the combination of chosen strategies $\left(s_{A}, s_{B}\right)$ is known, i.e., the utility functions mapping $\left(s_{A}, s_{B}\right)$ on a number [2]. The expected pay-off quantifies the preference of the players.

In these lecture notes the idea of identifying strategic moves with quantum operations as introduced in Refs. [6] ]] is further developed. This approach appears to be fruitful in at least two ways [6-9]. On the one hand several recently proposed applications of quantum information theory can already be conceived as competitive situations where several parties with more or less opposed motives interact. These parties may, for example, apply quantum operations on a bi-partite quantum system [10]. In the same context, quantum cloning has been formulated as a game between two players [11]. Similarly, eavesdropping in quantum cryptography [12] can be regarded as a game between the eavesdropper and the sender, and there are similarities of the extended form of quantum versions of games and quantum algorithms [13,14]. On the other hand a generalisation of the theory of decisions into the domain of quantum probabilities seems interesting, as the roots of game theory are partly in probability theory. In this context it is of interest to investigate what solutions are attainable if superpositions of strategies are allowed for [6,

Game theory does not explicitly concern itself with how the information is transmitted once a decision is taken. Yet, it should be clear that the practical implementation of any (classical) game inevitably makes use of the the exchange of voting papers, faxes, emails, ballots, and the like. In the Prisoners' Dilemma, e.g., the two parties have to communicate with an advocate by talking to her or by writing a short letter on which the decision is indicated. Bearing in mind that a game is also about the transfer of information, it becomes legitimate to ask what happens if these carriers of information are taken to be quantum systems, quantum information being a fundamental notion of information.

By classical means a two player binary choice game may be played as follows: An arbiter takes two coins and forwards one coin each to the players. The players then receive their coin with head up and may keep it as it is ("cooperate") or turn it upside down so that tails is up ("defection"). Both players then return the coins to the arbiter who calculates the players' final pay-off corresponding to the combination of strategies he obtains from the players. Here, the coins serve as the physical carrier of information in the game. In a quantum version of such a game quantum systems would be used as such carriers of information. For a binary choice two player game an implementation making use of minimal resources involves two qubits as physical carriers.

\section{QUANTUM GAMES AND QUANTUM STRATEGIES}

Any quantum system which can be manipulated by two parties or more and where the utility of the moves can be reasonably quantified, may be conceived as a quantum game.

A two-player quantum game $\Gamma=\left(\mathcal{H}, \rho, S_{A}, S_{B}, P_{A}, P_{B}\right)$ is completely specified by the underlying Hilbert space $\mathcal{H}$ of the physical system, the initial state $\rho \in$ $\mathcal{S}(\mathcal{H})$, where $\mathcal{S}(\mathcal{H})$ is the associated state space, the sets $S_{A}$ and $S_{B}$ of permissible quantum operations of the two players, and the utility functionals $P_{A}$ and $P_{B}$, which specify the utility for each player. A quantum strategy $s_{A} \in S_{A}, s_{B} \in S_{B}$ is a quantum operation, that is, a completely positive trace-preserving map mapping the state space on itself [15]. The quantum game's definition also includes certain implicit rules, such as the order of the implementation of the respective quantum strategies. Rules also exclude certain actions, as the alteration of the pay-off during the game. 
The quantum games proposed in Refs. [6], [ل], and [9] can be cast into this form. Also, the quantum cloning device as described in [11] can be said to be a quantum game in this sense. A quantum game is called zero-sum game, if the expected payoffs sum up to zero for all pairs of strategies, that is, if $P_{A}\left(s_{A}, s_{B}\right)=-P_{B}\left(s_{A}, s_{B}\right)$ for all $s_{A} \in S_{A}, s_{B} \in S_{B}$. Otherwise, it is called a non-zero sum game.

It is natural to call two quantum strategies of Alice $s_{A}$ and $s_{A}^{\prime}$ equivalent, if $P_{A}\left(s_{A}, s_{B}\right)=P_{A}\left(s_{A}^{\prime}, s_{B}\right)$ and $P_{B}\left(s_{A}, s_{B}\right)=P_{A}\left(s_{A}^{\prime}, s_{B}\right)$ for all possible $s_{B}$. That is, if $s_{A}$ and $s_{A}^{\prime}$ yield the same expected pay-off for both players for all allowed strategies of Bob. In the same way strategies $s_{B}$ and $s_{B}^{\prime}$ of Bob will be identified.

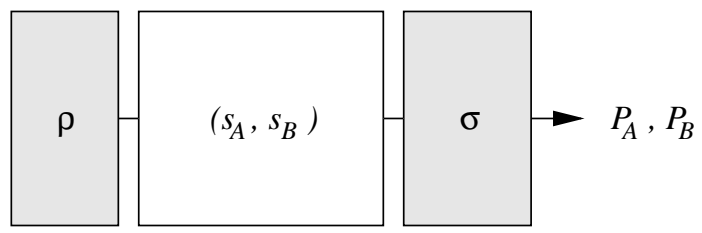

FIG. 2. The general setup of a quantum game.

A solution concept provides advice to the players with respect to the action they should take. The following solution concepts will be used in the remainder of this lecture. These definitions are fully analogous to the corresponding definitions in standard game theory [2].

A quantum strategy $s_{A}$ is called dominant strategy of Alice if

$$
P_{A}\left(s_{A}, s_{B}^{\prime}\right) \geq P_{A}\left(s_{A}^{\prime}, s_{B}^{\prime}\right)
$$

for all $s_{A}^{\prime} \in S_{A}, s_{B}^{\prime} \in S_{B}$. Analogously we can define a dominant strategy for Bob. A pair $\left(s_{A}, s_{B}\right)$ is said to be an equilibrium in dominant strategies if $s_{A}$ and $s_{B}$ are the players' respective dominant strategies. A combination of strategies $\left(s_{A}, s_{B}\right)$ is called a Nash equilibrium if

$$
\begin{aligned}
& P_{A}\left(s_{A}, s_{B}\right) \geq P_{A}\left(s_{A}^{\prime}, s_{B}\right), \\
& P_{B}\left(s_{A}, s_{B}\right) \geq P_{B}\left(s_{A}, s_{B}^{\prime}\right)
\end{aligned}
$$

for all $s_{A}^{\prime} \in S_{A}, s_{B}^{\prime} \in S_{B}$. A pair of strategies $\left(s_{A}, s_{B}\right)$ is called Pareto optimal, if it is not possible to increase one player's pay-off without lessening the pay-off of the other player.

A solution in dominant strategies is the strongest solution concept for a non-zero sum game. In the Prisoner's Dilemma defection is the dominant strategy, as it is favourable regardless what strategy the other party picks. Typically, however, the optimal strategy depends on the strategy chosen by the other party. A Nash equilibrium implies that neither player has a motivation to unilaterally alter his or her strategy from this equilibrium solution, as this action will lessen his or her pay-off. Given that the other player will stick to the strategy corresponding to the equilibrium, the best result is achieved by also playing the equilibrium solution. The concept of Nash equilibria is of paramount importance to studies of non-zero-sum games. It is, however, only an acceptable solution concept if the Nash equilibrium is unique. For games with multiple equilibria the application of a hierarchy of natural refinement concepts may finally eliminate all but one of the Nash equilibria. Note that a Nash equilibrium is not necessarily efficient. In the Prisoners' Dilemma for example there is a unique equilibrium, but it is not Pareto optimal, meaning that there is another outcome which would make both players better off.

\section{TWO-QUBIT QUANTUM GAMES}

In the subsequent investigation we turn to specific games where the classical version of the game is faithfully entailed in the quantum game. In a quantum version 
of a binary choice game two qubits are prepared by a arbiter in a particular initial state, the qubits are sent to the two players who have physical instruments at hand to manipulate their qubits in an appropriate manner. Finally, the qubits are sent back to the arbiter who performs a measurement to evaluate the pay-off. For such a bi-partite quantum game the system of interest is a quantum system with underlying Hilbert space $\mathcal{H}=\mathcal{H}_{A} \otimes \mathcal{H}_{B}, \mathcal{H}_{A}=\mathcal{H}_{B}=\mathbb{C}^{2}$, and associated state space $\mathcal{S}(\mathcal{H})$. Quantum strategies $s_{A}$ and $s_{B}$ of Alice and Bob are local quantum operations acting in $\mathcal{H}_{A}$ and $\mathcal{H}_{B}$ respectively [16]. That is, Alice and Bob are restricted to implement their respective quantum strategy $s_{A}$ and $s_{B}$ on their qubit only. In this step they may choose any quantum strategy that is included in the set of strategies $S$. They are both well aware of the set $S$, but they do not know which particular quantum strategy the other party would actually implement. As the application of both quantum strategies amounts to a map $s_{A} \otimes s_{B}: \mathcal{S}(\mathcal{H}) \rightarrow \mathcal{S}(\mathcal{H})$, after execution of the moves the system is in the state

$$
\sigma=\left(s_{A} \otimes s_{B}\right)(\rho) .
$$

Particularly important will be unitary operations $s_{A}$ and $s_{B}$. They are associated with unitary operators $U_{A}$ and $U_{B}$, written as $s_{A} \sim U_{A}$ and $s_{B} \sim U_{B}$. In this case the final state $\sigma$ is given by

$$
\sigma=\left(U_{A} \otimes U_{B}\right) \rho\left(U_{A} \otimes U_{B}\right)^{\dagger} .
$$

From now on both the sets of strategies of Alice and Bob and the pay-off functionals are taken to be identical, that is,

$$
S_{A}=S_{B}=S \quad \text { and } \quad P_{A}=P_{B}=P,
$$

such that both parties face the same situation.

The quantum game $\Gamma=\left(\mathbb{C}^{2} \otimes \mathbb{C}^{2}, \rho, S, S, P, P\right)$ can be played in the following way: The initial state $\rho$ is taken to be a maximally entangled state in the respective state space. In order to be consistent with Ref. [6] let $\rho=|\psi\rangle\langle\psi|$ with

$$
|\psi\rangle=(|00\rangle+i|11\rangle) / \sqrt{2},
$$

where the first entry refers to $\mathcal{H}_{A}$ and the second to $\mathcal{H}_{B}$. The two qubits are forwarded to the arbiter who performs a projective selective measurement on the final state $\sigma$ with Kraus operators $\pi_{C C}, \pi_{C D}, \pi_{D C}$, and $\pi_{D D}$, where

$$
\begin{array}{ll}
\pi_{C C}=\left|\psi_{C C}\right\rangle\left\langle\psi_{C C}\right|, & \left|\psi_{C C}\right\rangle=(|00\rangle+i|11\rangle) / \sqrt{2}, \\
\pi_{C D}=\left|\psi_{C D}\right\rangle\left\langle\psi_{C D}\right|, & \left|\psi_{C D}\right\rangle=(|01\rangle-i|10\rangle) / \sqrt{2}, \\
\pi_{D C}=\left|\psi_{D C}\right\rangle\left\langle\psi_{D C}\right|, & \left|\psi_{D C}\right\rangle=(|10\rangle-i|01\rangle) / \sqrt{2}, \\
\pi_{D D}=\left|\psi_{D D}\right\rangle\left\langle\psi_{D D}\right|, & \left|\psi_{D D}\right\rangle=(|11\rangle+i|00\rangle) / \sqrt{2} .
\end{array}
$$

According to the outcome of the measurement, a pay-off of $A_{C C}, A_{C D}, A_{D C}$, or $A_{D D}$ is given to Alice, Bob receives $B_{C C}, B_{C D}, B_{D C}$, or $B_{D D}$. The utility functionals, also referred to as expected pay-off of Alice and Bob, read

$$
\begin{aligned}
& P_{A}\left(s_{A}, s_{B}\right)=A_{C C} \operatorname{tr}\left[\pi_{C C} \sigma\right]+A_{C D} \operatorname{tr}\left[\pi_{C D} \sigma\right]+A_{D C} \operatorname{tr}\left[\pi_{D C} \sigma\right]+A_{D D} \operatorname{tr}\left[\pi_{D D} \sigma\right] \\
& P_{B}\left(s_{A}, s_{B}\right)=B_{C C} \operatorname{tr}\left[\pi_{C C} \sigma\right]+B_{C D} \operatorname{tr}\left[\pi_{C D} \sigma\right]+B_{D C} \operatorname{tr}\left[\pi_{D C} \sigma\right]+B_{D D} \operatorname{tr}\left[\pi_{D D} \sigma\right]
\end{aligned}
$$

It is important to note that the Kraus operators are chosen in such a way that the classical game is fully entailed in the quantum game: The classical strategies $C$ and $D$ are associated with particular unitary operations,

$$
C \sim\left(\begin{array}{ll}
1 & 0 \\
0 & 1
\end{array}\right), \quad D \sim\left(\begin{array}{cc}
0 & 1 \\
-1 & 0
\end{array}\right) .
$$


$C$ does not change the state at all, $D$ implements a "spin-flip". If both parties stick to these classical strategies, Eq. (8a) and Eq. (8b) guarantee that the expected pay-off is exactly the pay-off of the corresponding classical game defined by the numbers $A_{C C}, A_{C D}, A_{D C}, A_{D D}, B_{C C}, B_{C D}, B_{D C}$, and $B_{D D}$. E.g., if Alice plays $C$ and Bob chooses $D$, the state $\sigma$ after implementation of the strategies is given by

$$
\sigma=(C \otimes D)(\rho)=\left|\psi_{C D}\right\rangle\left\langle\psi_{C D}\right|,
$$

such that Alice obtains $A_{C D}$ units and Bob $B_{C D}$ units pay-off (see Fig. 1). In this way the peculiarities of strategic moves in the quantum domain can be adequately studied. The players may make use of additional degrees of freedom which are not available with randomisation of the classical strategies, but they can also stick to mere classical strategies. This scheme can be applied to any two player binary choice game and is to a high extent canonical.

\section{A. Prisoners' Dilemma}

We now investigate the solution concepts for the quantum analogue of the Prisoners' Dilemma (see Fig. 11) [17],

$$
\begin{aligned}
& A_{C C}=B_{C C}=3, \quad A_{D D}=B_{D D}=1, \\
& A_{C D}=B_{D C}=0, \quad A_{D C}=B_{C D}=5 .
\end{aligned}
$$

In all of the following sets of allowed strategies $S$ the classical options (to defect and to cooperate) are included. Several interesting sets of strategies and concomitant solution concepts will at this point be studied. The first three subsections involve local unitary operations only, while in the last subsection other quantum operations are considered as well.

\section{One-parameter set of strategies.}

The first set of strategies $S^{(C L)}$ involves quantum operations $s_{A}$ and $s_{B}$ which are local rotations with one parameter. The matrix representation of the corresponding unitary operators is taken to be

$$
U(\theta)=\left(\begin{array}{cc}
\cos (\theta / 2) & \sin (\theta / 2) \\
-\sin (\theta / 2) & \cos (\theta / 2)
\end{array}\right)
$$

with $\theta \in[0, \pi]$. Hence, in this simple case, selecting strategies $s_{A}$ and $s_{B}$ amounts to choosing two angles $\theta_{A}$ and $\theta_{B}$. The classical strategies of defection and cooperation are also included in the set of possible strategies, $C \sim U(0), D \sim U(\pi)$. An analysis of the expected pay-offs $P_{A}$ and $P_{B}$,

$$
\begin{aligned}
P_{A}\left(\theta_{A}, \theta_{B}\right) & =3\left|\cos \left(\theta_{A} / 2\right) \cos \left(\theta_{B} / 2\right)\right|^{2}+5\left|\cos \left(\theta_{B} / 2\right) \sin \left(\theta_{A} / 2\right)\right|^{2} \\
& +\left|\sin \left(\theta_{A} / 2\right) \sin \left(\theta_{B} / 2\right)\right|^{2}, \\
P_{B}\left(\theta_{A}, \theta_{B}\right) & =3\left|\cos \left(\theta_{A} / 2\right) \cos \left(\theta_{B} / 2\right)\right|^{2}+5\left|\sin \left(\theta_{B} / 2\right) \cos \left(\theta_{A} / 2\right)\right|^{2} \\
& +\left|\sin \left(\theta_{A} / 2\right) \sin \left(\theta_{B} / 2\right)\right|^{2},
\end{aligned}
$$

shows that this game is the classical Prisoners' Dilemma game [6]. The pay-off functions are actually identical to the analogous functions in the ordinary Prisoners' Dilemma with mixed (randomised) strategies, where cooperation is chosen with the classical probability $p=\cos ^{2}(\theta / 2)$. The inequalities

$$
\begin{aligned}
& P_{A}\left(D, s_{B}\right) \geq P_{A}\left(s_{A}, s_{B}\right), \\
& P_{B}\left(s_{A}, D\right) \geq P_{B}\left(s_{A}, s_{B}\right)
\end{aligned}
$$


hold for all $s_{A}, s_{B} \in S^{(C L)}$, therefore, $(D, D)$ is an equilibrium in dominant strategies and thus the unique Nash equilibrium. As explained in the introduction this equilibrium is far from being efficient, because $P_{A}(D, D)=P_{B}(D, D)=1$ instead of the Pareto optimal pay-off which would be 3.

\section{Two-parameter set of strategies}

A more general set of strategies is the following two-parameter set $S^{(T P)}$. The matrix representation of operators corresponding to quantum strategies from this set is given by

$$
U(\theta, \phi)=\left(\begin{array}{cc}
e^{i \phi} \cos (\theta / 2) & \sin (\theta / 2) \\
-\sin (\theta / 2) & e^{-i \phi} \cos (\theta / 2)
\end{array}\right)
$$

with $\theta \in[0, \pi]$ and $\phi \in[0, \pi / 2]$. Selecting a strategy $s_{A}, s_{B}$ then means choosing appropriate angles $\theta_{A}, \phi_{A}$ and $\theta_{B}, \phi_{B}$. The classical pure strategies can be realised as

$$
C \sim U(0,0) \text { and } \quad D \sim U(\pi, 0) .
$$

This case has also been considered in Ref. [6]. The expected pay-off for Alice, e.g., explicitly reads

$$
\begin{aligned}
& P_{A}\left(\theta_{A}, \phi_{A}, \theta_{B}, \theta_{B}\right)=3\left|\cos \left(\phi_{A}+\phi_{B}\right) \cos \left(\theta_{A} / 2\right) \cos \left(\theta_{B} / 2\right)\right|^{2} \\
+ & 5\left|\sin \left(\phi_{A}\right) \cos \left(\theta_{A} / 2\right) \sin \left(\theta_{B} / 2\right)-\cos \left(\phi_{B}\right) \cos \left(\theta_{B} / 2\right) \sin \left(\theta_{A} / 2\right)\right|^{2} \\
+ & \left|\sin \left(\phi_{A}+\phi_{B}\right) \cos \left(\theta_{A} / 2\right) \cos \left(\theta_{B} / 2\right)+\sin \left(\theta_{A} / 2\right) \sin \left(\theta_{B} / 2\right)\right|^{2} .
\end{aligned}
$$

It turns out that the previous Nash equilibrium $(D, D)$ of $S^{(C L)}$ is no longer an equilibrium solution, as both players can benefit from deviating from $D$. However, concomitant with the disappearance of this solution another Nash equilibrium has emerged, given by $(Q, Q)$. The strategy $Q$ is associated with a matrix

$$
Q \sim U(0, \pi / 2)=\left(\begin{array}{cc}
i & 0 \\
0 & -i
\end{array}\right)
$$

This Nash equilibrium is unique [6] and serves as the only acceptable solution of the game. The astonishing fact is that $P_{A}(Q, Q)=P_{B}(Q, Q)=3$ (instead of 1 ) so that the Pareto optimum is realised. No player could gain without lessening the other player's expected pay-off. In this sense one can say that the dilemma of the original game has fully disappeared. In the classical game only mutual cooperation is Pareto optimal, but this pair of strategies does not correspond to a Nash equilibrium.

\section{General unitary operations}

One can generalise the previous setting to the case where Alice and Bob can implement operations $s_{A}$ and $s_{B}$ taken from $S^{(G U)}$, where $S^{(G U)}$ is the set of general local unitary operations. Here, it could be suspected that the solution becomes more efficient the larger the sets of allowed operations are. But this is not the case. The previous Pareto optimal unique Nash equilibrium $(Q, Q)$ ceases to be an equilibrium solution if the set is enlarged: For any strategy $s_{B} \in S^{(G U)}$ there exists an optimal answer $s_{A} \in S^{(G U)}$ resulting in

$$
\left(s_{A} \otimes s_{B}\right)(\rho)=\left|\psi_{D C}\right\rangle\left\langle\psi_{D C}\right|,
$$


with $\rho$ given in Eq. (6). That is, for any strategy of Bob $s_{B}$ there is a strategy $s_{A}$ of Alice such that

$$
P_{A}\left(s_{A}, s_{B}\right)=5 \text { and } P_{B}\left(s_{A}, s_{B}\right)=0:
$$

Take

$$
s_{A} \sim\left(\begin{array}{ll}
a & b \\
c & d
\end{array}\right), s_{B} \sim\left(\begin{array}{cc}
-i b & a \\
-d & -i c
\end{array}\right)
$$

where $a, b, c, d$ are appropriate complex numbers. Given that Bob plays the strategy $s_{B}$ associated with a particular Nash equilibrium $\left(s_{A}, s_{B}\right)$, Alice can always apply the optimal answer $s_{A}$ to achieve the maximal possible pay-off. However, the resulting pair of quantum strategies can not be an equilibrium since again, the game being symmetric, Bob can improve his pay-off by changing his strategy to his optimal answer $s_{B}^{\prime}$. Hence, there is no pair $\left(s_{A}, s_{B}\right)$ of pure strategies with the property that the players can only lose from unilaterally deviating from this pair of strategies.

Yet, there remain to be Nash equilibria in mixed strategies which are much more efficient than the classical outcome of the equilibrium in dominant strategies $P_{A}(D, D)=P_{B}(D, D)=1$. In a mixed strategy of Alice, say, she selects a particular quantum strategy $s_{A}$ (which is also called pure strategy) from the set of strategies $S_{A}$ with a certain classical probability. That is, mixed strategies of Alice and Bob are associated with maps of the form

$$
\rho \longmapsto \sigma=\sum_{i, j} p_{A}^{(i)} p_{B}^{(j)}\left(U_{A}^{(i)} \otimes U_{B}^{(j)}\right) \rho\left(U_{A}^{(i)} \otimes U_{B}^{(j)}\right)^{\dagger},
$$

$p_{A}^{(i)}, p_{B}^{(i)} \in[0,1], i, j=1,2, \ldots, N$, with

$$
\sum_{i} p_{A}^{(i)}=\sum_{j} p_{B}^{(j)}=1
$$

$U_{A}^{(i)}$ and $U_{B}^{(j)}$ are local unitary operators corresponding to pure strategies $s_{A}^{(i)}$ and $s_{B}^{(j)}$.

The map given by Eq. 27) acts in $\mathcal{H}_{A}$ and $\mathcal{H}_{A}$ as a doubly stochastic map, that is, as a completely positive unital map [18]. As a result, the final reduced states $\operatorname{tr}_{B}[\sigma]$ and $\operatorname{tr}_{A}[\sigma]$ must be more mixed than the reduced initial states $\operatorname{tr}_{B}[\rho]$ and $\operatorname{tr}_{A}[\rho]$ in the sense of majorisation theory [19]. As the initial state $\rho$ is a maximally entangled state, all accessible states after application of a mixed strategy of Alice and Bob are locally identical to the maximally mixed state $1 / \operatorname{dim}\left(\mathcal{H}_{A}\right)=1 / \operatorname{dim}\left(\mathcal{H}_{B}\right)$, which is a multiple of 1.

The following construction, e.g., yields an equilibrium in mixed quantum strategies: Allow Alice to choose from two strategies $s_{A}^{(1)}$ and $s_{A}^{(2)}$ with probabilities $p_{A}^{(1)}=1 / 2$ and $p_{A}^{(2)}=1 / 2$, while Bob may take $s_{B}^{(1)}$ or $s_{B}^{(2)}$, with

$$
\begin{gathered}
s_{A}^{(1)} \sim\left(\begin{array}{ll}
1 & 0 \\
0 & 1
\end{array}\right), \quad s_{A}^{(2)} \sim\left(\begin{array}{cc}
-i & 0 \\
0 & i
\end{array}\right), \\
s_{B}^{(1)} \sim\left(\begin{array}{cc}
0 & 1 \\
-1 & 0
\end{array}\right), \quad s_{B}^{(2)} \sim\left(\begin{array}{cc}
0 & -i \\
-i & 0
\end{array}\right) .
\end{gathered}
$$

His probabilities are also given by $p_{B}^{(1)}=1 / 2$ and $p_{B}^{(2)}=1 / 2$. The quantum strategies of Eq. (24a) and Eq. (24b) are mutually optimal answers and have the property that

$$
\begin{array}{cl}
P_{A}\left(s_{A}^{(i)}, s_{B}^{(i)}\right)=0, & P_{B}\left(s_{A}^{(i)}, s_{B}^{(i)}\right)=5, \\
P_{A}\left(s_{A}^{(i)}, s_{B}^{(3-i)}\right)=5, & P_{B}\left(s_{A}^{(i)}, s_{B}^{(3-i)}\right)=0,
\end{array}
$$


for $i=1,2$. Due to the particular constraints of Eq. (25a) and Eq. (25b) there exists no other mixed strategy for Bob yielding a better pay-off than the above mixed strategy, given that Alice sticks to the equilibrium strategy. This can be seen as follows. Let Alice use this particular mixed quantum strategy as above and let Bob use any mixed quantum strategy

$$
s_{B}^{(1)}, \ldots, s_{B}^{(N)}
$$

together with $p_{A}^{(1)}, \ldots, p_{A}^{(N)}$. The final state $\sigma$ after application of the strategies is given by the convex combination

$$
\sigma=\sum_{i=1,2} \sum_{j} p_{A}^{(i)} p_{B}^{(j)}\left(s_{A}^{(i)} \otimes s_{B}^{(j)}\right)(\rho),
$$

This convex combination can only lead to a smaller expected pay-off for Bob than the optimal pure strategy $s_{B}^{(k)}$ in Eq. $(26), k \in\{1, \ldots, N\}$. Such optimal pure strategies are given by $s_{B}^{(1)}$ and $s_{B}^{(2)}$ as in Eq. (24b) leading to an expected pay-off for Bob of $P_{B}\left(s_{A}, s_{B}\right)=2.5$; there are no pure strategies which achieve a larger expected pay-off. While both pure strategies $s_{B}^{(1)}$ and $s_{B}^{(2)}$ do not correspond to an equilibrium, the mixed strategy where $s_{B}^{(1)}$ and $s_{B}^{(2)}$ are chosen with $p_{B}^{(1)}=1 / 2$ and $p_{B}^{(2)}=1 / 2$ actually does. Nash equilibria consist of pairs of mutually optimal answers, and only for this choice of Bob the original mixed quantum strategy of Alice is her optimal choice, as the same argument applies also to her, the game being symmetric.

This Nash equilibrium is however not the only one. There exist also other fourtuples of matrices than the ones presented in Eq. (24a) and Eq. (24b) that satisfy Eq. (25a) and Eq. (25b). Such matrices can be made out by appropriately rotating the matrices of Eq. (24a ) and Eq. (24b). In the light of the fact that there is more than one equilibrium it is not obvious which Nash equilibrium the players will realise. It is at first not even evident whether a Nash equilibrium will be played at all. But the game theoretical concept of the focal point effect [20,2] helps to resolve this issue.

To explore the general structure of any Nash equilibrium in mixed strategies we continue as follows: let

$$
U_{A}^{(1)}, \ldots, U_{A}^{(N)}
$$

together with $p_{A}^{(1)}, \ldots, p_{A}^{(N)}$ specify the mixed strategy pertinent to a Nash equilibrium of Alice. Then there exists a mixed strategy $U_{B}^{(1)}, \ldots, U_{B}^{(N)}, p_{B}^{(1)}, \ldots, p_{B}^{(N)}$ of Bob which rewards Bob with the best achievable pay-off, given that Alice plays this mixed strategy. Yet, the pair of mixed strategies associated with

$$
Q U_{A}^{(1)} Q^{\dagger}, \ldots, Q U_{A}^{(N)} Q^{\dagger}, \quad Q U_{B}^{(1)} Q^{\dagger}, \ldots, Q U_{B}^{(N)} Q^{\dagger}
$$

with $p_{A}^{(1)}, \ldots, p_{A}^{(N)}, p_{B}^{(1)}, \ldots, p_{B}^{(N)}$ is another Nash equilibrium. This equilibrium leads to the same expected pay-off for both players, and is fully symmetric to the previous one. Doubly applying $Q$ as $Q Q U_{A}^{(1)} Q^{\dagger} Q^{\dagger}, \ldots, Q Q U_{A}^{(N)} Q^{\dagger} Q^{\dagger}$ results again into a situation with equivalent strategies as the original ones. For a given Nash equilibrium as above the one specified by Eq. (29) will be called dual equilibrium.

However, there is a single Nash equilibrium $(R, R)$ which is the only one which gives an expected pay-off of $P_{A}(R, R)=P_{B}(R, R)=2.25$ and which is identical to its dual equilibrium: it is the simple map

$$
\rho \longmapsto \sigma=1 / \operatorname{dim}(\mathcal{H}) .
$$

Indeed, there exist probabilities $p_{A}^{(1)}, \ldots, p_{A}^{(N)}$ and unitary operators $U_{A}^{(1)}, \ldots, U_{A}^{(N)}$ such that $\sum_{i} p_{A}^{(i)}\left(U_{A}^{(i)} \otimes 1\right) \rho\left(U_{A}^{(i)} \otimes 1\right)^{\dagger}=1 / \operatorname{dim}(\mathcal{H})[19]$. If Alice has already selected 
$s_{A}=R$, the application of $s_{B}=R$ will not change the state of the quantum system any more.

Assume that Eq. (28) and Eq. (29) are associated with equivalent quantum strategies. This means that they have to produce the same expected pay-off for all quantum strategies $s_{B}$ of Bob. If Alice and Bob apply $s_{A} \otimes s_{B}$ they get an expected pay-off according to Eq. (8a) and Eq. (8b); if Alice after implementation of $s_{A}$ manipulates the quantum system by applying the local unitary operator $Q \otimes 1$, they obtain

$P_{A}^{\prime}\left(s_{A}, s_{B}\right)=A_{D D} \operatorname{tr}\left[\pi_{C C} \sigma\right]+A_{D C} \operatorname{tr}\left[\pi_{C D} \sigma\right]+A_{C D} \operatorname{tr}\left[\pi_{D C} \sigma\right]+A_{C C} \operatorname{tr}\left[\pi_{D D} \sigma\right]$,

$P_{B}^{\prime}\left(s_{A}, s_{B}\right)=B_{D D} \operatorname{tr}\left[\pi_{C C} \sigma\right]+B_{D C} \operatorname{tr}\left[\pi_{C D} \sigma\right]+B_{C D} \operatorname{tr}\left[\pi_{D C} \sigma\right]+B_{C C} \operatorname{tr}\left[\pi_{D D} \sigma\right]$.

The only $s_{A}$ with the property that $P_{A}^{\prime}\left(s_{A}, s_{B}\right)=P_{A}\left(s_{A}, s_{B}\right)$ and $P_{B}^{\prime}\left(s_{A}, s_{B}\right)=$ $P_{B}\left(s_{A}, s_{B}\right)$ for all $s_{B}$ is the map given by Eq. (30).

In principle, any Nash equilibrium may become a self-fulfilling prophecy if the particular Nash equilibrium is expected by both players. It has been pointed out that in a game with more than one equilibrium, anything that attracts the players' attention towards one of the equilibria may make them expect and therefore realise it [20]. The corresponding focal equilibrium [2] is the one which is conspicuously distinguished from the other Nash equilibria. In this particular situation there is indeed one Nash equilibrium different from all the others: it is the one which is equivalent to its dual equilibrium, the map which simply maps the initial state on the maximally mixed state. For all other expected pay-offs both players are ambivalent between (at least) two symmetric equilibria. The expected pay-off the players will receive in this focal equilibrium -

$$
P_{A}(R, R)=P_{B}(R, R)=2.25
$$

- is not fully Pareto optimal, but it is again much more efficient than the classically achievable outcome of 1 [21].

\section{Completely positive trace-preserving maps corresponding to local operations}

In this scenario both Alice and Bob may perform any operation that is allowed by quantum mechanics. That is, the set of strategies $S^{(C P)}$ is made up of $\left(s_{A}, s_{B}\right)$, where both $s_{A}$ and $s_{B}$ correspond to a completely positive trace-preserving map

$$
\left(s_{A} \otimes s_{B}\right)(\rho)=\sum_{i} \sum_{j}\left(A_{i} \otimes B_{j}\right) \rho\left(A_{i} \otimes B_{j}\right)^{\dagger}
$$

corresponding to a local operation, associated with Kraus operators $A_{i}$ and $B_{j}$ with $i, j=1,2, \ldots$. The trace-preserving property requires $\sum_{i} A_{i}^{\dagger} A_{i}=1$ and $\sum_{i} B_{i}^{\dagger} B_{i}=$ 1. This case has already been mentioned in Ref. [6]. The quantum strategies $s_{A}$ and $s_{B}$ do no longer inevitably act as unital maps in the respective Hilbert spaces as before. In other words, the reduced states of Alice and Bob after application of the quantum strategy are not necessarily identical to the maximally mixed state $1 / \operatorname{dim}\left(\mathcal{H}_{A}\right)$.

As already pointed out in Ref. [6], the pair of strategies $(Q, Q)$ of the twoparameter set of strategies $S^{(T P)}$ is again no equilibrium solution. It is straightforward to prove that the Nash equilibria of the type of Eq. (24a) and Eq. (24b) of mixed strategies with general local unitary operations are, however, still present, and each of these equilibria yields an expected pay-off of 2.5 .

In addition, as strategies do no longer have to be locally unital maps, it is not surprising that new Nash equilibria emerge: Alice and Bob may, e.g., perform a measurement associated with Kraus operators

$$
A_{1}=|0\rangle\left\langle 0\left|\quad A_{2}=\right| 1\right\rangle\left\langle 1\left|, \quad B_{1}=D\right| 0\right\rangle\left\langle 0\left|\quad B_{2}=D\right| 1\right\rangle\langle 1| .
$$


This operation yields a final state $\sigma=\left(s_{A} \otimes s_{B}\right)(\rho)=(|01\rangle\langle 01|+| 10\rangle\langle 10|) / 2$. Clearly neither Alice nor Bob can gain from unilaterally deviating from their strategy.

One can nevertheless argue as in the previous case. Again, all Nash equilibria occur at least in pairs. First, there are again the dual equilibria from $S^{(G U)}$. Second, there are Nash equilibria $\left(s_{A}, s_{B}\right), s_{A} \neq s_{B}$, with the property that $\left(s_{B}, s_{A}\right)$ is also a Nash equilibrium yielding the same expected pay-off. The only Nash equilibrium invariant under application of $Q$ and exchange of the strategies of the players is again $(R, R)$ defined in the previous subsection, which yields a pay-off $P_{A}(R, R)=P_{B}(R, R)=2.25$. This is the solution of the game is the most general case. While both players could in principle do better (as the solution lacks Pareto optimality), the efficiency of this focal equilibrium is much higher than the equilibrium in dominant strategies of the classical game. Hence, also in this most general case both players gain from using quantum strategies.

This study shows that the efficiency of the equilibrium the players can reach in this game depends on the actions the players may take. One feature, however is present in each of the considered sets: both players can increase their expected pay-offs drastically by resorting to quantum strategies.

\section{B. Chicken}

In the previous classical game - the Prisoners' Dilemma - an unambiguous solution can be specified consisting of a unique Nash equilibrium. However, this solution was not efficient, thus giving rise to the dilemma. The situation of the players in the Chicken game [2], 3],

$$
\begin{aligned}
& A_{C C}=B_{C C}=6, \quad A_{C D}=B_{D C}=8, \\
& A_{D C}=B_{C D}=2, \quad A_{D D}=B_{D D}=0,
\end{aligned}
$$

can be described by the matrix of Fig. 3 .

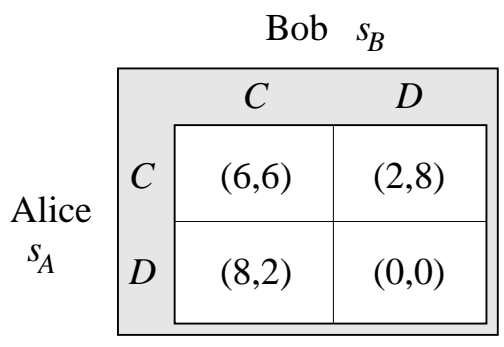

FIG. 3. The pay-off matrix of the so-called Chicken game.

This game has two Nash equilibria $(C, D)$ and $(D, C)$ : it is not clear how to anticipate what the players' decision would be. In addition to the two Nash equilibria in pure strategies there is an equilibrium in mixed strategies, yielding an expected pay-off 4 [2].

In order to investigate the new features of the game if superpositions of classical strategies are allowed for, three set of strategies are briefly discussed:

\section{One-parameter set of strategies}

Again, we consider the set of strategies $S^{(C L)}$ of one-dimensional rotations. The strategies $s_{A}$ and $s_{B}$ are associated with local unitary operators

$$
U(\theta)=\left(\begin{array}{cc}
\cos (\theta / 2) & \sin (\theta / 2) \\
-\sin (\theta / 2) & \cos (\theta / 2)
\end{array}\right)
$$


with $\theta \in[0, \pi]$,

$$
C \sim U(0)=\left(\begin{array}{ll}
1 & 0 \\
0 & 1
\end{array}\right), \quad D \sim U(\pi)=\left(\begin{array}{cc}
0 & 1 \\
-1 & 0
\end{array}\right) .
$$

Then as before, the quantum game yields the same expected pay-off as the classical game in randomised strategies. This means that still two Nash equilibria in pure strategies are present.

\section{Two-parameter set of strategies}

The players can actually take advantage of an additional degree of freedom which is not accessible in the classical game. If they may apply unitary operations from $S^{(T P)}$ of the type

$$
U(\theta, \phi)=\left(\begin{array}{cc}
e^{i \phi} \cos (\theta / 2) & \sin (\theta / 2) \\
-\sin (\theta / 2) & e^{-i \phi} \cos (\theta / 2)
\end{array}\right)
$$

with $\theta \in[0, \pi]$ and $\phi \in[0, \pi / 2]$ the situation is quite different than with $S^{(C L)}$. $(C, D)$ and $(C, D)$ with $C \sim U(0,0)$ and $D \sim U(\pi, 0)$ are no longer equilibrium solutions. E.g., given that $s_{A}=D$ the pair of strategies $(D, Q)$ with $Q \sim U(0, \pi / 2)$ yields a better expected pay-off for Bob than $(D, C)$, that is to say $P_{B}(D, Q)=8$, $P_{B}(D, C)=2$. In fact $(Q, Q)$ is now the unique Nash equilibrium with $P_{A}(Q, Q)=$ $P_{B}(Q, Q)=6$, which follows from an investigation of the actual expected payoffs of Alice and Bob analogous to Eq. (17). This solution is not only the unique acceptable solution of the game, but it is also an equilibrium that is Pareto optimal. This contrasts very much with the situation in the classical game, where the two equilibria were not that efficient.

\section{Completely positive trace-preserving maps corresponding to local operations}

As in the considerations concerning the Prisoner's Dilemma game, more than one Nash equilibrium is present, if both players can take quantum strategies from the set $S^{(C P)}$, and all Nash equilibria emerge at least in pairs as above. The focal equilibrium is given by $(R, R)$, resulting in a pay-off of $P_{A}(R, R)=P_{B}(R, R)=4$, which is the same as the mixed strategy of the classical game.

\section{SUMMARY AND CONCLUSION}

In these lecture notes the idea of implementing quantum operations as strategic moves in a game is explored [6]. In detail, we investigated games which could be conceived as a generalisation into the quantum domain of a two player binary choice game. As a toy model for more complex scenarios we studied quantum games where the efficiency of the equilibria attainable when using quantum strategies could be contrasted with the efficiency of solutions in the corresponding classical game. We investigated a hierarchy of quantum strategies $S^{(C L)} \subset S^{(T P)} \subset S^{(G U)} \subset S^{(C P)}$. Again [6, [7], we found superior performance of quantum strategies as compared to classical strategies.

The nature of a game is determined by the rules of the game. In particular, the appropriate solution concept depends on the available strategic moves. Obviously, a player cannot make a meaningful choice without knowing the options at his or her disposal. So it comes to no surprise that also the actual achievable pay-off in such a game depends on the set of allowed strategies. Roughly speaking, one can 
say that the possibility of utilising strategies which are not feasible in the analogous classical game implicates a significant advantage. In the models studied in detail two kinds of "dilemmas" were "resolved": (i) On the one hand there are quantum games with an efficient unambiguous solution, while in the classical analogue only an inefficient equilibrium can be identified. By taking advantage of appropriate quantum strategies much more efficient equilibria could be reached. In certain sets of strategies even a maximally efficient solution - the Pareto optimum - was attainable. (ii) On the other hand, there exist quantum games with a unique solution with a classical equivalent which offers two Nash equilibria of the same quality.

This paper deals with simple set-ups in which information is exchanged quantum-mechanically. The emphasis was to examine how situations where strategies are identified with quantum operations applied on quantum mechanical carriers of information are different from the classical equivalent. It is the hope that these investigations may enable us to better understand competitive structures in a game theoretical sense in applications of quantum information theory.

\section{ACKNOWLEDGEMENTS}

We would like to thank Maciej Lewenstein, Onay Urfalıoglu, Joel Sobel, Tom Cover, Charles H. Bennett, Martin B. Plenio, and Uta Simon for helpful suggestions. We also acknowledge fruitful discussions with the participants of the A2 Consortial Meeting. This work was supported by the European Union and the DFG.

[1] J. von Neumann and O. Morgenstern, The Theory of Games and Economic Behaviour (Princeton University Press, Princeton, 1947).

[2] R.B. Myerson, Game Theory: An Analysis of Conflict (MIT Press, Cambridge, 1991).

[3] W. Poundstone, Prisoners' Dilemma. John von Neumann, Game Theory, and the Puzzle of the Bomb (Doubleday, New York, 1992).

[4] M.D. Davis, Game Theory. A Nontechnical Introduction (Dover, New York, 1970).

[5] A.W. Tucker, unpublished (1950).

[6] J. Eisert, M. Wilkens, and M. Lewenstein, Phys. Rev. Lett. 83, 3077 (1999).

[7] D. Meyer, Phys. Rev. Lett. 82, 1052 (1999).

[8] P. Ball, Nature, Science Update 991021-3 (1999); G.P. Collins, Scientific American, January issue (2000).

[9] L. Goldenberg, L. Vaidman, and S. Wiesner, Phys. Rev. Lett. 82, 3356 (1999).

[10] M.B. Plenio and V. Vedral, Contemp. Phys. 39, 431 (1998).

[11] R.F. Werner, Phys. Rev. A 58, 1827 (1998).

[12] C.H. Bennett and G. Brassard, in Proceedings of the IEEE International Conference on Computers, Systems, and Signal Processing, Bangalore, India (IEEE, New York, 1984); C.H. Bennett, F. Bessette, G. Brassard, L. Salvail, and J. Smolin, J. Crypto. 5, 3 (1992).

[13] P.W. Shor, SIAM J. Comput. 26, 1484 (1997).

[14] A. Ekert, Phys. Rev. Lett. 67, 661 (1991).

[15] E.g., Alice may allow a coupling of the original quantum system to an auxiliary quantum system and let the two unitarily interact. After performing a projective measurement on the composite system she could eventually consider the original system again by taking a partial trace with respect to the auxiliary part.

[16] The quantum strategies $s_{A} \otimes 1$ and $1 \otimes s_{B}$ are in the following identified with $s_{A}$ and $s_{B}$, respectively.

[17] From a game theoretical viewpoint, any positive numbers satisfying the symmetry conditions $A_{C C}=B_{C C}, A_{D D}=B_{D D}, A_{C D}=B_{D C}, A_{D C}=B_{C D}$ and the inequalities 
$A_{D C}>A_{C C}>A_{D D}>A_{C D}$ and $A_{C C} \geq\left(A_{C D}+A_{D C}\right) / 2$ define a (strong) Prisoners' Dilemma.

[18] P.M. Alberti and A. Uhlmann, Stochasticity and Partial Order: Doubly Stochastic Maps and Unitary Mixing (VEB Deutscher Verlag der Wissenschaften, Berlin, 1982).

[19] A.W. Marshall and I. Olkin, Inequalities: Theory of Majorisation and its Applications (Academic Press, New York, 1979);

[20] T.C. Schelling, The Strategy of Conflict (Harvard University Press, Cambridge (MA), 1960).

[21] In the classical game both players could also play $C$ and $D$ with probabilities $p=1 / 2$ yielding the same expected pay-off of 2.25 for both players, but this pair of mixed strategies would be no equilibrium solution, as any player could benefit from simply choosing the dominant strategy $D$. 